\title{
Up-Regulation of Pain Behavior and Glial Activity in the Spinal Cord after Compression and Application of Nucleus Pulposus onto the Sciatic Nerve in Rats
}

\author{
Masaki Norimoto, Yoshihiro Sakuma, Miyako Suzuki, Sumihisa Orita, Kazuyo Yamauchi, \\ Gen Inoue, Yasuchika Aoki, Tetsuhiro Ishikawa, Masayuki Miyagi, Hiroto Kamoda, \\ Gou Kubota, Yasuhiro Oikawa, Kazuhide Inage, Takeshi Sainoh, Jun Sato, \\ Junichi Nakamura, Tomoaki Toyone, Kazuhisa Takahashi, Seiji Ohtori \\ Department of Orthopaedic Surgery, Graduate School of Medicine, Chiba University, Chiba, Japan
}

\begin{abstract}
Study Design: Experimental animal study.
Purpose: To evaluate pain-related behavior and changes in glial activity in the spinal dorsal horn after combined sciatic nerve compression and nucleus pulposus (NP) application in rats.

Overview of Literature: Mechanical compression and inflammation caused by prostaglandins and cytokines at disc herniation sites induce pain. Structural changes and pain-associated cytokines in the dorsal root ganglia and spinal dorsal horn contribute to prolonged pain. Glial cells in the spinal dorsal horn may also function in pain transmission.

Methods: The sciatic nerve was compressed with NP for 2 seconds using forceps in the NP+nerve compression group; the shamoperated group received neither compression nor NP; and the control group received no operation. Mechanical hyperalgesia was measured for 3 weeks using von Frey filaments. Glial activity in the spinal dorsal horn was examined 7 days and 14 days postsurgery using anti-glial fibrillary acidic protein and anti-lonized calcium binding adaptor molecule-1 antibodies to detect astrocytes and microglia, respectively.

Results: Mechanical hyperalgesia was detected throughout the 14-day observation in the NP+nerve compression group, but not in control or sham-operated groups $(p<0.05)$. Both astrocytes and microglia were significantly increased in the spinal dorsal horn of the $\mathrm{NP}+$ nerve compression group compared to control and sham groups on days 7 and $14(p<0.05)$.

Conclusions: Nerve compression with NP application produces pain-related behavior, and up-regulates astrocytes and microglia in the spinal dorsal horn, suggesting that these glia may be related to pain transmission.
\end{abstract}

Keywords: Rat; Pain; Nerve; Glia; Spinal cord

\section{Introduction}

Radicular pain is a common symptom of lumbar disc herniation; it is caused by mechanical compression and inflammation of nerve roots in animals and humans $[1,2]$. Cytokines, generated at sites of mechanical compres-

Received Sep 22, 2013; Revised Nov 14, 2013; Accepted Nov 17, 2013

Corresponding author: Seiji Ohtori

Department of Orthopaedic Surgery, Graduate School of Medicine, Chiba University,

1-8-1 Inohana, Chuo-ku, Chiba 260-8670, Japan

Tel: +81-43-226-2117, Fax: +81-43-226-2116, E-mail: sohtori@faculty.chiba-u.jp 
sion and inflammation, produce pain in response to lumbar disc herniation [3].

Several studies have reported increases in prostaglandins, pain-associated neuropeptides such as substance $\mathrm{P}$ (SP) and calcitonin gene-related peptide (CGRP), cytokines such as tumor necrosis factor alpha (TNFa), and ion channels in the dorsal root ganglion (DRG) neurons and spinal dorsal horn in animal models of disc herniation. This suggests that primary afferents mediate pain after disc herniation via these neuropeptides and cytokines in DRG neurons and the spinal dorsal horn [4-7].

In addition to the factors described above, glial cells such as astrocytes and microglia are also activated after nerve injury and inflammation in the central nervous system $[8,9]$. Glial cells release proinflammatory cytokines that induce proliferation of other glial cells, and the upregulation of cytokines is associated with nerve degeneration in the central nervous system $[10,11]$. Recent reports have demonstrated the relationship between pain and glial activity in the central nervous system; glial activity after nerve injury and inflammation produces hyperalgesia and allodynia $[12,13]$. This activation of glial cells is thought to be involved in the pathogenesis of neuropathic and inflammatory pain.

While many studies have reported glial activation in peripheral neuropathic and inflammatory pain models, there have been few reports on spinal glial activation in a lumbar disc herniation model. The purpose of the current study was to evaluate pain-related behavior and changes in astrocytes and microglial activity in the spinal dorsal horn after combined sciatic nerve compression and nucleus pulposus (NP) application in rats.

\section{Materials and Methods}

All protocols for animal procedures were approved by the Ethics Committees of Chiba University in accordance with the National Institutes of Health Guidelines for the Care and Use of Laboratory Animals (1996 revision).

\section{Harvesting NP from lumbar intervertebral discs of donor rats}

Ten 6-week-old male Sprague Dawley rats were anesthetized with intraperitoneal (i.p.) injections of sodium pentobarbital (40 mg/kg). NP was harvested from lumbar intervertebral discs (L2/3 to L4/5) and was used in the following experiments.

\section{Models}

Sixty 6-week-old male Sprague Dawley rats were anesthetized with sodium pentobarbital (40 mg/kg, i.p.), and their left sciatic nerves were exposed. NP was applied to the sciatic nerve and compressed once for 2 seconds with a 2-mm-wide smooth forceps ( $\mathrm{NP}+$ nerve compression group; $n=20$ ), as described previously [4]. The sciatic nerve was compressed gradually, and the compression was released if the rat exhibited a cramp in its hind paw. In sham-operated rats (sham-operated group, $n=20$ ), the left sciatic nerves were exposed, but the sciatic nerves were not pinched and NP was not applied. Another group of rats (control group, $n=20$ ) received no surgery and were used as controls. Of the 60 rats used in these studies, 30 were used only for the assessment of pain behavior, and 30 rats were used only for immunohistochemical analysis.

\section{Evaluation of pain behavior}

Tactile hyperalgesia was evaluated in 10 rats each from the control, sham-operated, and NP+nerve compression groups. Mechanical pain thresholds of the left hind paw were assessed using von Frey filaments with a bending force of $1.20 \mathrm{~g}$ (noxious stimulation). The von Frey filament was applied to the plantar surface of the paw for five trials. The responses to these stimuli were scored as follows: "0", no response; "1", withdrawal from the von Frey filament; and "2", immediate flinching or licking of the hind paw as previously reported [14]. The scores from the five trials in each animal were added to give a total score, from 0 to 10 for each animal; the average nociceptive score was calculated for each group: mean nociceptive score $=\Sigma$ total score for each animal/number of animals.

\section{Immunohistochemistry for glial fibrillary acidic pro- tein (astrocytes) and Iba-1 (microglia) in the spinal dorsal horn}

10 rats each were evaluated from the control, shamoperated, and $\mathrm{NP}+$ nerve compression groups. At 7 days (each group, $n=5$ ) and 14 days (each group, $n=5$ ) postsurgery, rats were anesthetized with sodium pentobarbital (40 mg/kg, i.p.) and perfused transcardially with $250 \mathrm{~mL}$ of $4 \%$ paraformaldehyde in phosphate buffer $(0.1 \mathrm{M}, \mathrm{pH}$ 
7.4). The level of the epiconus was easily detected form the ventral aspect, and the spinal cord at the epiconus corresponding to the L5 spinal segments was resected. The specimens were immersed in the same fixative solution overnight at $4^{\circ} \mathrm{C}$. After storing in $0.01 \mathrm{M}$ phosphate buffer saline (PBS) containing 20\% sucrose for 20 hours at $4{ }^{\circ} \mathrm{C}$, the lumbar spinal cord segments were sectioned at $40 \mu \mathrm{m}$ thickness with a cryostat.

Sections of the lumbar segments were collected in PBS. The specimens were then treated for 90 minutes in blocking solution, 0.01 MPBS containing $0.3 \%$ Triton X-100, and $1 \%$ normal goat serum at room temperature. Tissue was processed for immunohistochemistry with a mouse antibody to glial fibrillary acidic protein (GFAP) (1:1000; Sigma, St. Louis, MO, USA) or a rabbit antibody to Ionized calcium binding adaptor molecule-1 (Iba-1) (1:1000; Wako, Osaka, Japan) using the free-floating technique; tissue was incubated in primary antibodies for 20 hours at $4{ }^{\circ} \mathrm{C}$, followed by incubation with goat anti-rabbit Alexa 488-fluorescein-conjugated antibody (1:400; Molecular Probes, Eugene, OR, USA).

Sections were mounted on poly-L-lysine-coated slides. The sections were examined using a fluorescence microscope (Nikon, Tokyo, Japan). The number of GFAP or
Iba-1 immunoreactive (IR) cells in 5 sections from each segment were counted at $\times 400$ magnification using a counting grid. The number of GFAP or Iba-1-IR cells per $0.0225 \mathrm{~mm}^{2}$ in laminae I-IV in the spinal dorsal horn was quantitatively evaluated in a blinded manner. Identification of spinal cord layers was made according to the classification by Rexed [15].

\section{Statistical analysis}

Hind paw withdrawal latencies were compared using one-way analysis of variance (ANOVA) for repeated measurements. For multiple comparisons, we used Dunnett's post-hoc test. Comparison of GFAP or Iba-1 immunoreactivity among groups was made using a one-way ANOVA followed by Dunnett's post-hoc test. Values of $p<0.05$ were considered statistically significant; error bars indicate standard error of the mean.

\section{Results}

\section{Mechanical hyperalgesia}

Fig. 1 shows pain behavior in the 3 groups. The control

(Average score)

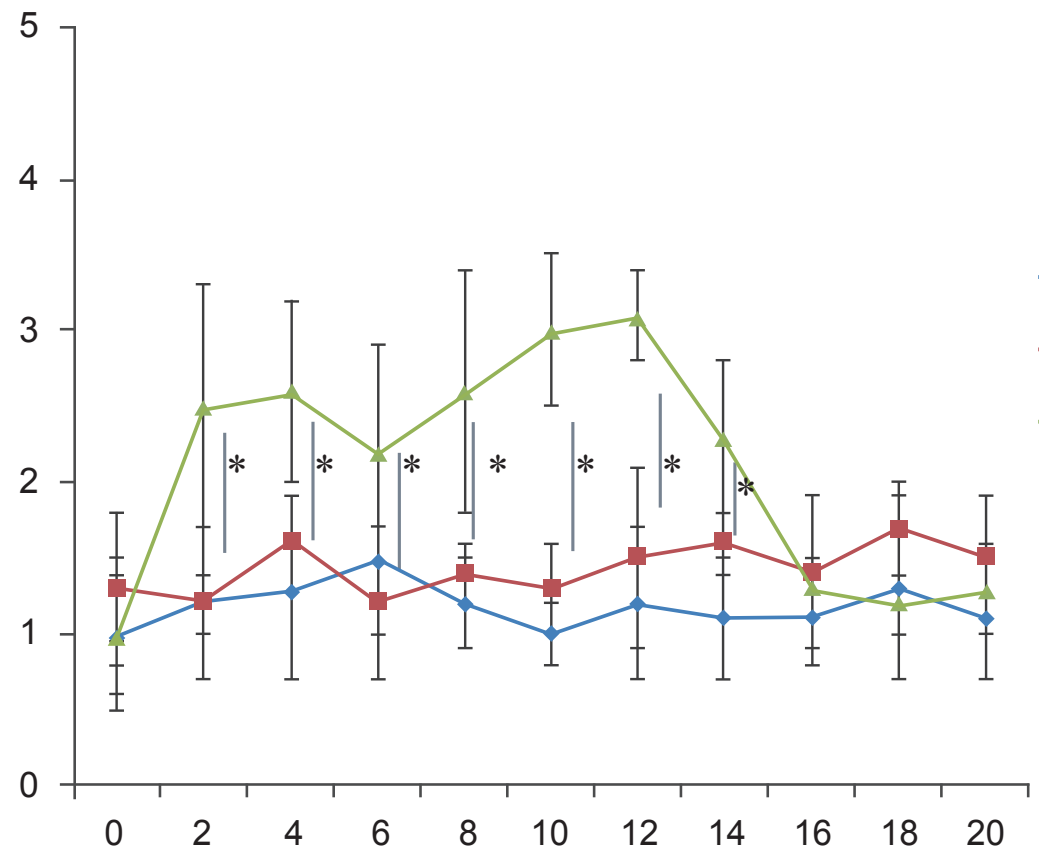

$\rightarrow$ Control

$\rightarrow$ Sham

$\rightarrow \mathrm{NP}+$ compression

$* p<0.05$

Fig. 1. Comparison of mechanical hyperalgesia among the control, sham-operated, and nucleus pulposus (NP)+compression groups. Data are shown as mean \pm standard error of the mean. High scores indicate an increased level of pain. 
and sham-operated groups did not show pain behavior during the 3 weeks of monitoring. There was no significant difference in the pain scores of the control and sham-operated groups during the experimental period. The NP+nerve compression group showed pain behavior during the 2 week observation period, and the pain score was significantly higher than that of the control and sham-operated groups $(p<0.05)$.

\section{GFAP or Iba-1 immunoreactivity in the spinal dorsal horn}

Fig. 2 shows GFAP or Iba- 1 immunoreactivity in the spinal dorsal horn. In control and sham-operated groups, GFAP or Iba-1 immunoreactivity was not observed in the spinal dorsal horn. In contrast, GFAP and Iba-1 immunoreactivity was observed in the spinal dorsal horn in the $\mathrm{NP}+$ nerve compression group.

Fig. 3 shows a comparison of GFAP immunoreactivity in the spinal dorsal horn among the 3 groups. The number of GFAP-IR astrocytes was significantly higher in the $\mathrm{NP}+$ nerve compression group compared with control and sham-operated rats on postsurgical days 7 and 14 $(p<0.05)$ (Fig. 3).

Fig. 4 shows a comparison of Iba- 1 immunoreactivity in the spinal dorsal horn among the 3 groups. The number of Iba-1-IR microglia was significantly higher in the
$\mathrm{NP}+$ nerve compression group compared with control and sham-operated rats on postsurgical days 7 and 14 $(p<0.05)$ (Fig. 4).

\section{Discussion}

Our results showed that nerve compression with NP application produced pain-related behavior and upregulated GFAP-IR astrocytes and Iba-1-IR microglia in the spinal dorsal horn. These findings suggest that nerve compression with NP application may induce glial activity in the spinal cord, and these activated glia may play a crucial role in pain transmission in the spinal dorsal horn.

Two studies using animal models have reported on the relationship between glial activity in the spinal cord and lumbar disc herniation $[6,16]$. Park et al. [16] reported that autologous implantation of NP on the left L5 nerve root, just proximal to the dorsal root ganglion, induces thermal hyperalgesia and mechanical allodynia, and leads to an up-regulation of chemokines and their receptors in spinal neurons and glia. Ito et al. [6] reported that autologous implantation of NP on the left L5 nerve root activated p38 mitogen-activated protein kinases in spinal microglia and that microglia may play an important role in the pathophysiology of pain following lumbar disc herniation. In the current study, we used a rat model of sciatic nerve compression with the application of NP. Our
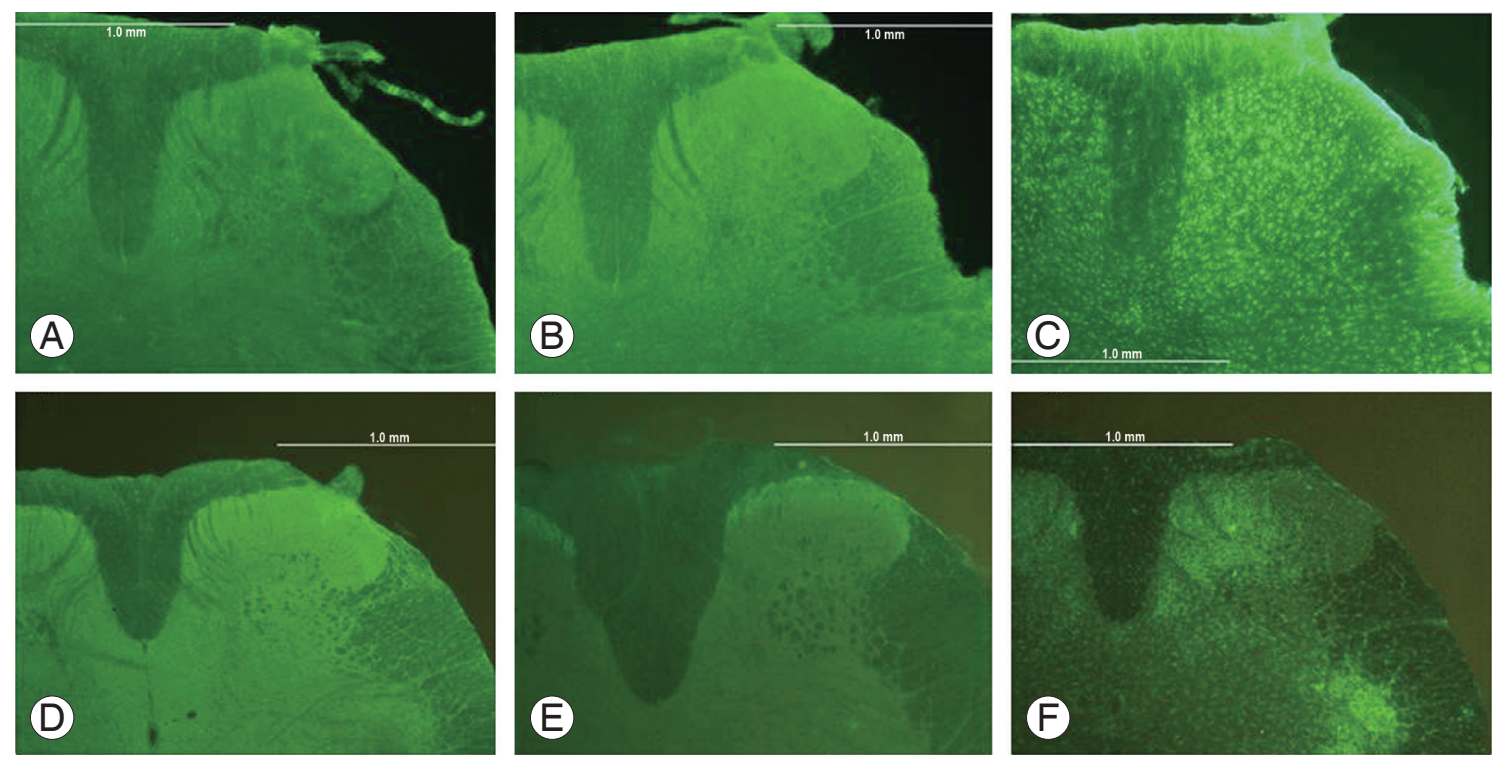

Fig. 2. GFAP-IR astrocytes in the spinal dorsal horn of control (A), sham-operated (B), and NP+compression (C) groups on postsurgical day 14. Iba-1-IR microglia in the spinal dorsal horn of control (D), sham-operated (E), and NP+compression (F) groups on postsurgical day 14. GFAP, glial fibrillary acidic protein; IR, immunoreactive; NP, nucleus pulposus; Iba-1, lonized calcium binding adaptor molecule-1. 
results demonstrated pain-related behavior in this model, and the up-regulation of GFAP-IR astrocytes and Iba-1IR microglia in the spinal dorsal horn. The up-regulation of glial activity observed in our model is consistent with previous reports.

Recently, it has been reported that the effects of NP on nerve roots were closely associated with cytokines such as TNFa, and cyclooxygenase-2 (COX-2) $[7,17]$. In animal

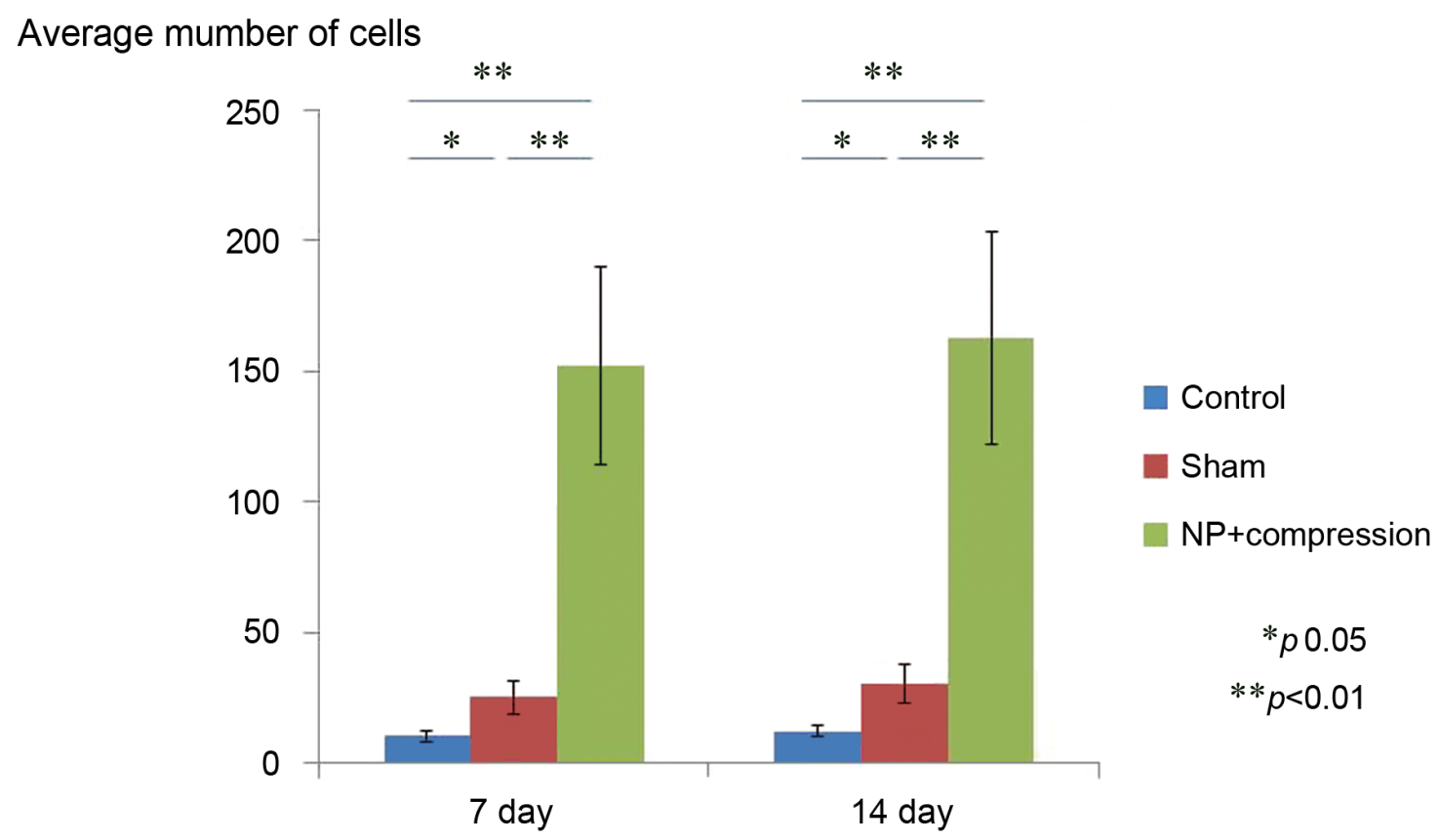

Fig. 3. The average number of GFAP-IR astrocytes in the spinal dorsal horn of control, sham-operated, and NP+compression groups on day 7 and 14. Data are shown as mean \pm standard error of the mean. GFAP, glial fibrillary acidic protein; IR, immunoreactive; NP, nucleus pulposus.

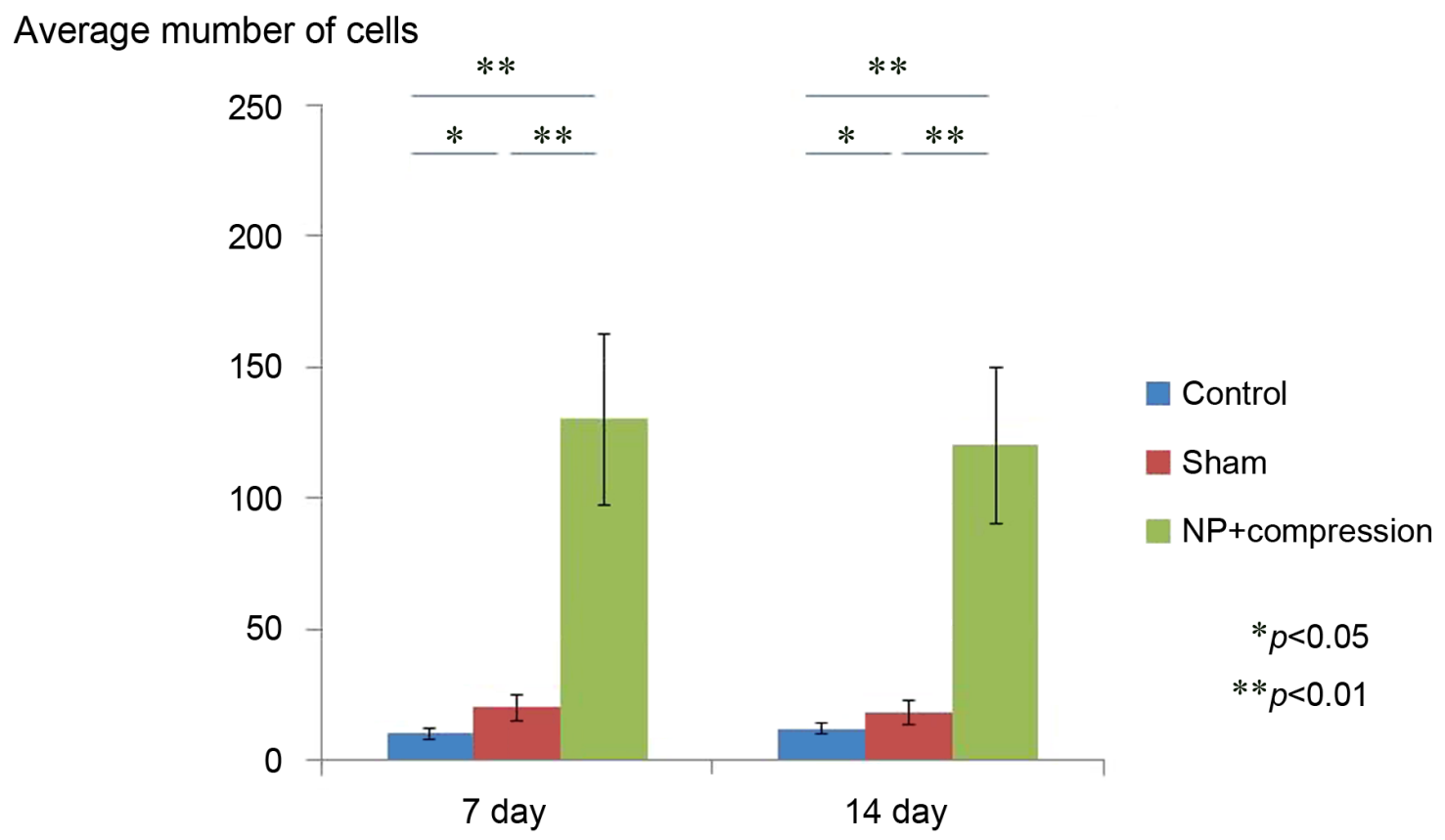

Fig. 4. The average number of Iba-1-IR microglia in the spinal dorsal horn of control, sham-operated, and NP+compression groups on day 7 and 14. Data are shown as mean \pm standard error of the mean. Iba-1, lonized calcium binding adaptor molecule-1; IR, immunoreactive; NP, nucleus pulposus. 
lumbar disc herniation models demonstrating pain behavior, it was shown that nerve growth factor and brainderived neurotrophic factor (BDNF), which are related to inflammatory pain, as well as neuropeptides such as SP and CGRP and TNFa, are activated in the spinal cord and DRG [4,18-21]. TNFa induces the production of inflammatory neuropeptides, SP and CGRP, and induces the release of SP and CGRP in the spinal dorsal horn from the peripheral terminals [22-24]. Application of NP onto nerve roots was shown to induce a marked increase in BDNF which was released in the superficial layer of the dorsal horn in a disc herniation model $[19,20]$. In this model, treatment with infliximab, which is a TNFa inhibitor, reduced BDNF release in the spinal cord [20]. Taking these studies into consideration, we hypothesize that nerve compression plus NP application may influence glial activity in the spinal dorsal horn via the release of neuropeptides, growth factors, and cytokines from primary sensory afferents.

TNFa is the most widely used therapeutic target for radicular pain originating from lumbar disc herniation or spinal stenosis. A recent clinical study found that the TNFa inhibitor infliximab was effective in reducing sciatic nerve pain caused by lumbar disc herniation, and patients experienced no significant side effects [25]. Similarly, results from our clinical studies indicate that the epidural administration of a TNFa inhibitor onto the spinal nerve produces relief of radicular pain with no adverse effects [26]. Thus, TNFa inhibitors may be useful for the treatment of radicular pain caused by spinal stenosis [26].

The specific mechanisms by which central inflammatory cytokines exert their effects are not entirely understood, but the temporal delay in CNS activation after peripheral nerve injury suggests that retrograde axonal transport may be involved. TNF $\alpha$ at injured nerve sites is retrogradely transported to the spinal dorsal horn where TNFa is released from primary sensory terminals onto the spinal dorsal horn [27]. TNFa is known to affect the proliferation of astrocytes [10], and it has been suggested that its indirect role in triggering microglial and astroglial cell proliferation promotes scar formation [28]. However, it has also been reported that microglial and astroglial cells constitutively express type $1 \mathrm{TNF} \alpha$ receptors, suggesting that TNFa has the potential to directly affect them as well $[29,30]$. Combined, these data suggest a relationship between TNF $\alpha$ released from microglia and astrocytes in the spinal cord and neuropathic pain. In clinical applications, TNF $\alpha$ inhibitors administered to the spinal cord may be useful for the treatment of radicular pain caused by lumbar disc herniation.

It should be noted that there are some limitations in the current study. For example, we used a rat model of sciatic nerve compression plus inflammation by NP, although in patients with lumbar disc herniation the spinal nerve root is compressed by NP, not the sciatic nerve. However, the sciatic nerve compression model used in our study has been well-validated $[4,21,31]$. In these studies pain behavior was associated with the up-regulation of TNFa and CGRP in DRG neurons $[4,21,31]$. In view of the similarity between these previous reports and the pain-related behavior and cytokine expression observed in our study, we conclude that the model of sciatic nerve compression with NP application is a valid disc-herniation model. Second, we did not use NP only or nerve compression only groups in the current study. Here we report only on a sciatic nerve pinch model in rat; in this model, rats show mechanical and thermal hyperalgesia for 8 days and an increase in pain-associated neuropeptides [32]. Kawakami et al. [33] have reported that allografting NP onto the spinal nerve root without other treatment induced pain behavior for 1 week, and increased interleukin 1 around the spinal nerve roots. They have also reported that the allograft of NP only at the spinal nerve roots induced pain behavior for 1 week, and increased phospholipase A2 and nitric oxide around the spinal nerve roots [34]. In addition, mechanical compression and the effects of NP on the lumbar nerve root have been examined in rats. Silk loops placed around the nerve root resulted in thermal hyperalgesia only in rats which had NP applied to the nerve root [35]. In the current study, the NP with nerve compression group showed a longer period of pain behavior compared with previous reports, which investigated the effects of either NP only or nerve compression only. We hypothesize that while the application of NP only or nerve compression only can induce pain and glial activation in the spinal cord, nerve compression with NP application may induce a greater increase in glial activation in the spinal cord, and this difference may be more comparable to disc herniation pain than the individual effects of NP only or nerve compression only. However, further study will be needed to strengthen our hypothesis. Third, in the current study, the pain score returned to an insignificant range after 2 weeks, while the average cell count for astrocytes and microglia in the nerve compression+NP 
application group remained significantly higher than in the sham operated and control groups. We have previously reported the induction of pain behavior and glial activation or increased COX-2 in the spinal dorsal horn $[6,7]$. In the disc herniation model used in those studies, the application of NP to the nerve root induced mechanical allodynia in rats for 2 weeks; however, the increased COX-2 expression in the spinal dorsal horn was only observed for 1 week [7]. In contrast, application of NP to the nerve root in rats resulted in allodynia for 8 days, while microglial activation in the spinal dorsal horn was observed for more than 2 weeks [6]. Pain is generally transmitted from the periphery to the brain via the dorsal root ganglion and the spinal cord; thus several sites contribute to pain sensation. In order to clarify the discrepancy between the duration of pain and the duration of glial activation in the spinal dorsal horn observed in the current study. Further studies will be needed that examine changes in the dorsal root ganglion and brain.

\section{Conclusions}

In conclusion, the combination of sciatic nerve-compression with the application of NP produced pain-related behavior. The up-regulation of GFAP-IR astrocytes and Iba1-IR microglia was observed in the spinal dorsal horn. These findings suggest that nerve compression plus NP application may induce glial activity in the spinal cord, and that these glia may be related to pain transmission.

\section{Conflict of Interest}

No potential conflict of interest relevant to this article was reported.

\section{References}

1. Olmarker K, Rydevik B, Nordborg C. Autologous nucleus pulposus induces neurophysiologic and histologic changes in porcine cauda equina nerve roots. Spine (Phila Pa 1976) 1993;18:1425-32.

2. Toyone T, Takahashi K, Kitahara H, Yamagata M, Murakami M, Moriya H. Visualisation of symptomatic nerve roots. Prospective study of contrast-enhanced MRI in patients with lumbar disc herniation. J Bone Joint Surg Br 1993;75:529-33.

3. Olmarker K, Storkson R, Berge OG. Pathogenesis of sciatic pain: a study of spontaneous behavior in rats exposed to experimental disc herniation. Spine (Phila Pa 1976) 2002;27:1312-7.

4. Norimoto M, Ohtori S, Yamashita M, et al. Direct application of the TNF-alpha inhibitor, etanercept, does not affect CGRP expression and phenotypic change of DRG neurons following application of nucleus pulposus onto injured sciatic nerves in rats. Spine (Phila Pa 1976) 2008;33:2403-8.

5. Ohtori S, Inoue G, Koshi T, et al. Up-regulation of acid-sensing ion channel 3 in dorsal root ganglion neurons following application of nucleus pulposus on nerve root in rats. Spine (Phila Pa 1976) 2006;31: 2048-52.

6. Ito T, Ohtori S, Inoue $\mathrm{G}$, et al. Glial phosphorylated p38 MAP kinase mediates pain in a rat model of lumbar disc herniation and induces motor dysfunction in a rat model of lumbar spinal canal stenosis. Spine (Phila Pa 1976) 2007;32:159-67.

7. Ohtori S, Takahashi K, Aoki Y, et al. Spinal neural cyclooxygenase- 2 mediates pain caused in a rat model of lumbar disk herniation. J Pain 2004;5:385-91.

8. Wagner R, Myers RR. Schwann cells produce tumor necrosis factor alpha: expression in injured and noninjured nerves. Neuroscience 1996;73:625-9.

9. Watkins LR, Martin D, Ulrich P, Tracey KJ, Maier SF. Evidence for the involvement of spinal cord glia in subcutaneous formalin induced hyperalgesia in the rat. Pain 1997;71:225-35.

10. Selmaj KW, Farooq M, Norton WT, Raine CS, Brosnan CF. Proliferation of astrocytes in vitro in response to cytokines. A primary role for tumor necrosis factor. J Immunol 1990;144:129-35.

11. Stoll G, Jander S. The role of microglia and macrophages in the pathophysiology of the CNS. Prog Neurobiol 1999;58:233-47.

12. Fenzi F, Benedetti MD, Moretto G, Rizzuto N. Glial cell and macrophage reactions in rat spinal ganglion after peripheral nerve lesions: an immunocytochemical and morphometric study. Arch Ital Biol 2001; 139:357-65.

13. Zhou XF, Rush RA, McLachlan EM. Differential expression of the $\mathrm{p} 75$ nerve growth factor receptor in glia and neurons of the rat dorsal root ganglia after peripheral nerve transection. J Neurosci 1996;16: 2901-11.

14. Takasaki I, Andoh T, Shiraki K, Kuraishi Y. Allodynia 
and hyperalgesia induced by herpes simplex virus type-1 infection in mice. Pain 2000;86:95-101.

15. Rexed B. The cytoarchitectonic organization of the spinal cord in the cat. J Comp Neurol 1952;96:414-95.

16. Park HW, Ahn SH, Kim SJ, et al. Changes in spinal cord expression of fractalkine and its receptor in a rat model of disc herniation by autologous nucleus pulposus. Spine (Phila Pa 1976) 2011;36:E753-60.

17. Olmarker K, Larsson K. Tumor necrosis factor alpha and nucleus-pulposus-induced nerve root injury. Spine (Phila Pa 1976) 1998;23:2538-44.

18. Murata Y, Onda A, Rydevik B, Takahashi K, Olmarker K. Distribution and appearance of tumor necrosis factor-alpha in the dorsal root ganglion exposed to experimental disc herniation in rats. Spine (Phila $\mathrm{Pa}$ 1976) 2004;29:2235-41.

19. Obata K, Tsujino H, Yamanaka H, et al. Expression of neurotrophic factors in the dorsal root ganglion in a rat model of lumbar disc herniation. Pain 2002;99: 121-32.

20. Onda A, Murata Y, Rydevik B, Larsson K, Kikuchi S, Olmarker K. Infliximab attenuates immunoreactivity of brain-derived neurotrophic factor in a rat model of herniated nucleus pulposus. Spine (Phila Pa 1976) 2004;29:1857-61.

21. Yamashita M, Ohtori S, Koshi T, et al. Tumor necrosis factor-alpha in the nucleus pulposus mediates radicular pain, but not increase of inflammatory peptide, associated with nerve damage in mice. Spine (Phila Pa 1976) 2008;33:1836-42.

22. Ding M, Hart RP, Jonakait GM. Tumor necrosis factoralpha induces substance $\mathrm{P}$ in sympathetic ganglia through sequential induction of interleukin- 1 and leukemia inhibitory factor. J Neurobiol 1995;28:445-54.

23. Hua XY, Chen P, Fox A, Myers RR. Involvement of cytokines in lipopolysaccharide-induced facilitation of CGRP release from capsaicin-sensitive nerves in the trachea: studies with interleukin-1beta and tumor necrosis factor-alpha. J Neurosci 1996;16:4742-8.

24. Opree A, Kress M. Involvement of the proinflammatory cytokines tumor necrosis factor-alpha, IL-1 beta, and IL- 6 but not IL-8 in the development of heat hyperalgesia: effects on heat-evoked calcitonin generelated peptide release from rat skin. J Neurosci 2000; 20:6289-93.

25. Korhonen T, Karppinen J, Malmivaara A, et al. Efficacy of infliximab for disc herniation-induced sci- atica: one-year follow-up. Spine (Phila Pa 1976) 2004; 29:2115-9.

26. Ohtori S, Miyagi M, Eguchi Y, et al. Epidural administration of spinal nerves with the tumor necrosis factor-alpha inhibitor, etanercept, compared with dexamethasone for treatment of sciatica in patients with lumbar spinal stenosis: a prospective randomized study. Spine (Phila Pa 1976) 2012;37:439-44.

27. Shubayev VI, Myers RR. Anterograde TNF alpha transport from rat dorsal root ganglion to spinal cord and injured sciatic nerve. Neurosci Lett 2002;320:99101.

28. Merrill JE. Effects of interleukin-1 and tumor necrosis factor-alpha on astrocytes, microglia, oligodendrocytes, and glial precursors in vitro. Dev Neurosci 1991;13:130-7.

29. Kinouchi K, Brown G, Pasternak G, Donner DB. Identification and characterization of receptors for tumor necrosis factor-alpha in the brain. Biochem Biophys Res Commun 1991;181:1532-8.

30. Ohtori S, Takahashi K, Moriya H, Myers RR. TNFalpha and TNF-alpha receptor type 1 upregulation in glia and neurons after peripheral nerve injury: studies in murine DRG and spinal cord. Spine (Phila Pa 1976) 2004;29:1082-8.

31. Sakuma T, Kamoda H, Miyagi M, et al. Comparison of CatWalk analysis and von Frey testing for pain assessment in a rat model of nerve crush plus inflammation. Spine (Phila Pa 1976) 2013;38:E919-24.

32. Hirose K, Iwakura N, Orita S, et al. Evaluation of behavior and neuropeptide markers of pain in a simple, sciatic nerve-pinch pain model in rats. Eur Spine J 2010;19:1746-52.

33. Kawakami M, Tamaki T, Hashizume H, Weinstein JN, Meller ST. The role of phospholipase A2 and nitric oxide in pain-related behavior produced by an allograft of intervertebral disc material to the sciatic nerve of the rat. Spine (Phila Pa 1976) 1997;22:1074-9.

34. Kawakami M, Tamaki T, Weinstein JN, Hashizume H, Nishi H, Meller ST. Pathomechanism of pain-related behavior produced by allografts of intervertebral disc in the rat. Spine (Phila Pa 1976) 1996;21:2101-7.

35. Kawakami M, Tamaki T, Hayashi N, et al. Mechanical compression of the lumbar nerve root alters painrelated behaviors induced by the nucleus pulposus in the rat. J Orthop Res 2000;18:257-64. 